DOI: http://dx.doi.org/10.19177/reen.v14e12021120-146

\title{
FATORES QUE IMPACTAM NA DEPENDÊNCIA E COMPORTAMENTO DE COMPRA DE SMARTPHONES
}

\section{FACTORS THAT IMPACT ON SMARTPHONES DEPENDENCE AND PURCHASE PERFORMANCE \\ FACTORES QUE IMPACTAN EN LA DEPENDENCIA Y COMPORTAMIENTO DE COMPRA DE SMARTPHONES}

Railana Morais de Freitas

Bacharela em Administração pelo Instituto Federal Farroupilha

E-mail: railanamdef@gmail.com

Mariangela Amaral e Silva

Mestra em Integração Latino-Americana

Professora na Instituto Federal de Educação, Ciência e Tecnologia Farroupilha (IFFar)

E-mail: mariangela.silva@iffarroupilha.edu.br

\section{Ana Claudia da Rosa}

Doutoranda em Administração pela Universidade Federal de Santa Maria

Professora na Instituto Federal de Educação, Ciência e Tecnologia Farroupilha (IFFar)

E-mail: ana.claudiadarosaa@gmail.com

Artigo recebido em 15/07/2019. Revisado por pares em 04/09/2020. Reformulado em 06/09/2020. Recomendado para publicação em 15/03/2021, por Ademar Dutra (Editor Científico). Publicado em 30/04/2021. Avaliado pelo Sistema double blind review.

(C) Copyright 2021 UNISUL-PPGA/Revista Eletrônica de Estratégia \& Negócios. Todos os direitos reservados. Permitida citação parcial, desde que identificada a fonte. Proibida a reprodução total.

Revisão gramatical, ortográfica e ABNT de responsabilidade dos autores. 


\section{RESUMO}

A pesquisa caracterizou-se como quantitativa e descritiva, baseando-se no modelo de Ting et al. (2011), formado pelos construtos: conveniência, influência e necessidades sociais, dependência e comportamento de compra. O objetivo geral foi investigar o impacto dos fatores conveniência, necessidades e influências sociais na dependência do uso de smartphones, bem como o impacto dessa dependência de uso no comportamento de compra de smartphones em acadêmicos de um Instituto Federal. Os dados foram obtidos por meio da aplicação de questionários. Evidenciou-se que a conveniência e influências sociais impactam na dependência de smartphone e a dependência de uso impacta no comportamento de compra.

Palavras-chave: Smartphones; Dependência de uso; Comportamento de compra; Marketing de serviços.

\section{ABSTRACT}

The research was characterized as quantitative and descriptive, based on the model of Ting et al. (2011), formed by the constructs: convenience, influence and social needs, dependence and buying behavior. The overall objective was to investigate the impact of convenience factors, needs and social influences on the dependence on smartphone use, as well as the impact of this use dependence on smartphone buying behavior in academics of a Federal Institute. The data were obtained through the application of questionnaires. It has been shown that convenience and social influences impact on the dependence of smartphone and the dependence of use impact on the buying behavior.

Keywords: Smartphones; User dependence; Behavior of purchase; Marketing services.

\section{RESUMEN}

La investigación se caracterizó como cuantitativa y descriptiva, basándose en el modelo de Ting et al. (2011), formado por los constructos: conveniencia, influencia y necesidades sociales, dependencia y comportamiento de compra. El objetivo general fue investigar el impacto de los factores conveniencia, necesidades e influencias sociales en la dependencia del uso de Smartphone, así como el impacto de esa dependencia de uso en el comportamiento de compra de smartphone en académicos de un Instituto Federal. Los datos se obtuvieron mediante la aplicación de cuestionarios. Se evidenció que la conveniencia e influencias sociales impactan en la dependencia de smartphone y la dependencia de uso impacta en el comportamiento de compra.

Palabras-clave: Smartphones; Dependencia de uso; Comportamiento de compra; Marketing de servicios. 


\section{INTRODUÇÃO}

Com o avanço tecnológico e o avanço da internet, os smartphones ganharam novas funcionalidades e, principalmente, novos conceitos. Conquistaram espaço no dia a dia de seus usuários em função de sua praticidade e, em decorrência disso, o número de aparelhos em uso vem aumentando gradativamente ao longo dos anos.

De acordo com dados de uma pesquisa da Fundação Getúlio Vargas (FGV, 2018), o Brasil está na lista dos países que possuem os maiores números de vendas de telefones inteligentes nos últimos anos, que é como são conhecidos os smartphones, sendo a quantidade atual no Brasil acima de 220 milhões de aparelhos ativos. Conforme a mesma notícia, em dezembro de 2017, o país havia registrado um total de 210 milhões de aparelhos ativos.

Conforme aponta o Instituto Brasileiro de Geografia e Estatística (IBGE, 2018), a Pesquisa Nacional por Amostra de Domicílios Contínua (PNAD), realizada no quarto trimestre de 2016, com foco nos aspectos de acesso à internet e à televisão e posse de telefone móvel celular, revelou que em apenas 5,4\% dos domicílios particulares permanentes do país não havia qualquer tipo de telefone e em $97,2 \%$ dos 48.070 mil domicílios em que havia acesso à internet, o telefone móvel celular era utilizado para esse fim.

No âmbito do marketing de serviços nas organizações, Corrêa e Caon (2002) afirmam que serviços prestados com qualidade e excelência levam a empresa à obtenção de lucro e atuam na diferenciação do pacote de valor ofertado pelo mercado, gerando vantagem competitiva em relação aos seus concorrentes. Cobra (2014) também relata que a qualidade no serviço executado é um fator determinante na satisfação ou insatisfação do consumidor.

Diante da notável popularização desses aparelhos, da dependência das tecnologias móveis na vida de seus usuários, da importância do marketing na competitividade das organizações e da relevância comprovada sobre o tema, conforme dados expostos nesta introdução sobre o crescimento do uso dos smartphones no Brasil, sendo um mercado atual e em constante expansão, constatou-se a necessidade de uma maior e mais profunda 
compreensão dos fatores que impactam na dependência e no comportamento de compra dos smartphones.

Inserido nesta temática, o presente estudo caracterizou-se como uma pesquisa quantitativa e descritiva, tendo como base o modelo teórico proposto por Ting et al. (2011), o qual é formado pelos construtos: conveniência, influência social, necessidades sociais, dependência de uso e comportamento de compra de smartphones. Sendo assim, o objetivo geral da pesquisa foi investigar o impacto dos fatores Conveniência, Necessidades e Influências Sociais na dependência do uso de smartphones, bem como o impacto dessa dependência de uso no comportamento de compra de smartphones em acadêmicos de um Instituto Federal localizado na região sul do Brasil.

Com o intuito de atingir o objetivo geral e a sua complementação, os objetivos específicos deste estudo foram: a) identificar o perfil dos acadêmicos; b) adaptar e validar o modelo de Ting et al. (2011) para o contexto brasileiro; c) testar o impacto e verificar se as necessidades sociais, influência social e conveniência (variáveis independentes) são antecedentes da dependência de uso de smartphone (variável mediadora); d) verificar o impacto da dependência de uso (variável mediadora) no comportamento de compra (variável dependente).

Portanto, salienta-se a contribuição prática do estudo, o qual pode servir de subsídio para a área de marketing das empresas do segmento de telefonia móvel, auxiliando os gestores na compreensão de como a conveniência, as necessidades e as influências sociais afetam a dependência de uso, e como essa dependência afeta o comportamento de compra. Além disso, destaca-se a contribuição teórica, que ocorrerá por meio da adaptação e da validação do modelo proposto por Ting et al. (2011) para o contexto brasileiro, trazendo uma importante contribuição para a ciência da Administração, ao passo que este modelo ainda não foi aplicado e analisado no contexto nacional.

\section{REFERENCIAL TEÓRICO}

Neste tópico é apresentado o embasamento teórico da atual pesquisa. 


\subsection{MARKETING DE SERVIÇOS: TELEFONIA MÓVEL}

O setor de serviços ocupa posição de destaque em todos os países desenvolvidos, fato este evidenciado pela participação do setor na ocupação de mão de obra e na geração de riqueza refletida pelo Produto Interno Bruto (PIB) (CORRÊA; CAON, 2002). Para Grewal e Levy (2012, p. 228), serviço é "qualquer oferta intangível que envolve um ato, desempenho ou esforço que não pode ser fisicamente possuído; benefícios intangíveis para os clientes que são produzidos por pessoas ou máquinas e não podem ser separadas do produtor".

O setor de serviços é dividido em cinco categorias de oferta de mercado: bens puramente tangíveis, bens tangíveis associados a serviços, híbridos, serviço principal associado a bens ou serviços secundários e serviço puro. Os smartphones encontram-se alocados na categoria dos híbridos, caracterizados como ofertas igualmente compostas por bens e serviços. Ou seja, os consumidores compram o smartphone tanto pelos benefícios físicos do aparelho quanto pelos serviços ofertados pela marca e pela operadora, por exemplo (KOTLER; KELLER, 2012).

Conforme Cobra (2014, p. 86), “as mudanças tecnológicas nos produtos são altamente estimulantes para o consumo. O novo modelo de computador, a nova televisão digital ou mudanças em eletrônicos provoca ansiedade de consumo", e, assim, os consumidores passam a sentir necessidade de mudar seus produtos atuais por produtos com recursos tecnológicos mais avançados. Lemos (2007, p. 25) avalia as múltiplas funções dos smartphones e considera-os como Dispositivos Híbridos Móveis de Conexão Multirredes, como pode ser observado a seguir:

\footnotetext{
o que chamamos de telefone celular é um Dispositivo (um artefato, uma tecnologia de comunicação); Híbrido, já que congrega funções de telefone, computador, máquina fotográfica, câmera de vídeo, processador de texto, GPS, entre outras; Móvel, isto é, portátil e conectado em mobilidade funcionando por redes sem fio digitais, ou seja, de Conexão; e Multirredes, já que pode empregar diversas redes, como Bluetooth [...], internet (Wi-Fi ou Wi-Max) e redes de satélites para uso como dispositivo GPS (LEMOS, 2007, p. 25).
}

Cobra (2014, p. 220) ressalta que as tecnologias móveis estão amplamente difundidas em nossa sociedade e cita que "nos primórdios, a tecnologia tinha como objetivo ampliar a produtividade e racionalizar custos, e a conquista de mercado era um fator secundário, até Revista Eletrônica de Estratégia \& Negócios, Florianópolis, v.14, n. 1, jan./abr. 2021. 
mesmo um subproduto. Hoje é fator chave de sucesso". Sendo assim, os serviços prestados aos clientes, tanto os cobráveis quanto os não-cobráveis, fornecem à empresa meios eficientes de competição. Portanto, os gestores devem compreender que, quando é ofertada uma variedade adequada de serviços, como um adicional ao produto central, a organização consegue alcançar um desempenho mais eficaz do que os concorrentes (GRÖNROOS, 2009).

Um dos fatores relevantes na venda de um produto ou na prestação de um serviço é a compreensão do comportamento dos consumidores. De acordo com Churchill e Peter (2010, p. 146), "consumidores são pessoas que compram bens e serviços para si mesmas ou para outros, e não para fazer alguma outra coisa como revendê-los ou usá-los como insumos". Assim, para que as organizações atendam às necessidades desses consumidores e gerem lucro para si mesmas, é preciso compreender por que eles realizam determinadas compras e outras não.

Segundo Las Casas (2017), para o lançamento de um produto, torna-se necessário entender a razão pela qual os indivíduos realizam suas compras. Devido a essa necessidade, foram desenvolvidos estudos e teorias que envolvem esforços da psicologia, da sociologia, da economia e da antropologia, no intuito de interpretar os desejos dos diversos grupos de consumidores, compreendendo o funcionamento do processo de compra. Para isso, Churchill e Peter (2010) complementam que é preciso realizar estudos sobre os sentimentos, pensamentos, ações e influências que determinam mudanças sobre eles.

As necessidades de compra são desenvolvidas por um período de longa duração, que possivelmente faz com que os consumidores não tenham plena consciência de seus motivos para realizar ou não a compra, e essa decisão pode ser complicada pelo nível de percepção de necessidade atribuído pelo consumidor. Sendo assim, são identificados três níveis de percepção de necessidade: consciente, pré-consciente e inconsciente (FUTRELL, 2014).

No nível consciente, o consumidor tem plena certeza de suas necessidades e, dessa forma, a venda é realizada de uma maneira mais facilitada, pois ele está disposto a falar sobre suas necessidades, especificando exatamente o que deseja. Já no pré-consciente, os consumidores podem não estar com suas necessidades completamente desenvolvidas em Revista Eletrônica de Estratégia \& Negócios, Florianópolis, v.14, n. 1, jan./abr. 2021. 
suas mentes, o que dificulta o entendimento sobre qual é o desejo a ser satisfeito. E, no nível inconsciente, o consumidor não tem consciência dos motivos que o levam a comprar determinado produto, mas efetuam a compra mesmo assim (FUTRELL, 2014).

\subsection{MODELO TEÓRICO E HIPÓTESES DA PESQUISA}

Inserido no contexto de comportamento de compra, Ting et al. (2011) afirmaram que, para verificar o comportamento de compra dos consumidores, deve-se analisar os estímulos que afetam a dependência do uso de smartphones. Nessa perspectiva, esses autores identificaram como antecedentes da dependência de uso de smartphones as dimensões Conveniência, Influência e Necessidades sociais, e após a dependência como mediadora do comportamento de compra desses aparelhos.

Assim, os autores desenvolveram escalas para mensurar os fatores e aplicaram o estudo com 358 estudantes de universidades da Malásia. Além disso, uma adaptação do modelo de Ting et al. (2011) também foi aplicada no estudo de Suki (2013) com 200 estudantes universitários de outra universidade federal na Malásia.

Portanto, no presente estudo, foi utilizada uma adaptação do modelo proposto por Ting et al. (2011), formado por cinco construtos: conveniência, necessidades sociais, influências sociais, dependência de uso e comportamento de compra. A seguir, são apresentadas as hipóteses (Figura 1) e a definição dos construtos propostos pela pesquisa.

Figura 1 - Hipóteses da pesquisa

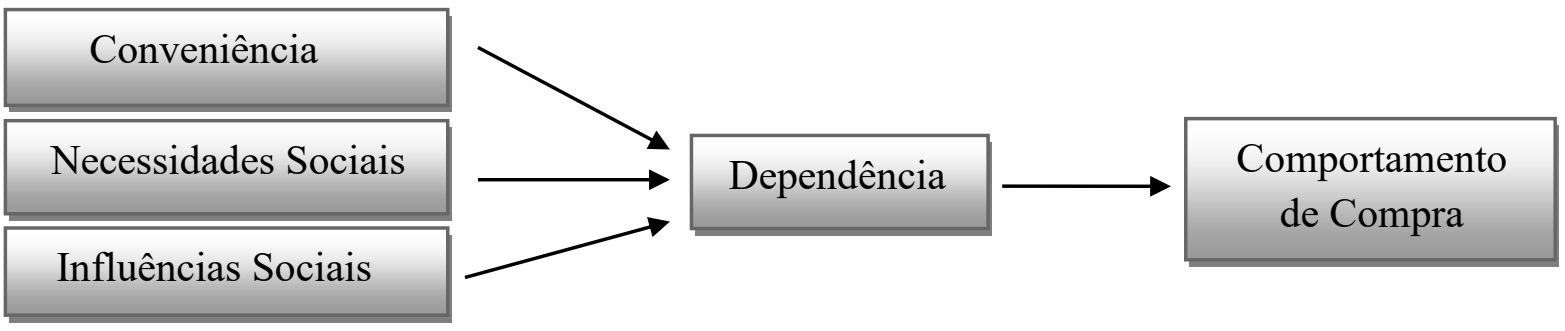

Fonte: Adaptado de Ting et al. (2011).

Com o avanço da internet, os usuários desta tecnologia desenvolveram características de dependência em relação a aparelhos que permitem conectar-se a ela. Considerando o uso constante dos smartphones, estudos vêm sendo realizados para um 
melhor entendimento dessa dependência tecnológica (RIBEIRO; SILVA, 2015; PICON et al. 2015; ROCHA et al. 2015).

Para Ribeiro e Silva (2015), as gerações mais novas são as mais tendenciosas ao uso de tecnologias como smartphones e tablets. Já a geração de mais idade, normalmente faz o uso de celulares simples, apenas com recursos para envio de Short Message Service (SMS), para efetuar ligações, sem aplicativos ou acesso à internet.

Nesse âmbito, destaca-se também que o uso excessivo dessas tecnologias pode ocasionar uma dependência tecnológica, que, segundo Picon et al. (2015), pode ser definida como um conjunto de transtornos em que seus usuários se tornam incapazes de controlar o uso dessas tecnologias, até mesmo quando este uso excessivo esteja causando efeitos negativos em sua da vida pessoal, profissional, mental, etc. Rocha et al. (2015) afirmam que existe um grupo de síndromes modernas em pauta na área da psicologia, que estuda certas implicações negativas causadas pelo uso abusivo de internet, e destaca a "nomofobia" (o medo absurdo de sair sem celular).

Para Picon et al. (2015), a chamada "nomofobia" (proveniente da expressão em inglês "no mobile phobia"), nome científico para a dependência de smartphone, faz referência à ansiedade, ao desconforto e ao estresse causado no usuário quando está longe de seu aparelho. Os sintomas da nomofobia se assemelham aos de outras dependências, como a abstinência, a tolerância e a saliência. Outro risco significativo relacionado ao uso frequente dos smartphones é a possibilidade de causar acidentes, como quedas, acidentes de trânsito e outros ocasionados em decorrência do desvio de atenção.

Uma pesquisa realizada por Borges e Joia (2013) constatou que a dependência de smartphone pode ser frequente também em profissionais. Por meio da pesquisa, que analisou as ações realizadas a partir do smartphone dos pesquisados, percebeu-se a prática constante de envio e/ou respostas de e-mails de assuntos profissionais durante os fins de semana. Tais dados confirmam que o smartphone proporciona independência ao usuário, mas também resulta em certa dependência, se o uso for muito frequente. 
Os autores Mok et al. (2014) realizaram um estudo relacionando o vício em internet e smartphone e as características dos usuários viciados. De acordo com os autores, o uso frequente de smartphones pode fazer com que seus usuários desenvolvam problemas de comportamento, tais como dificuldade adaptativa, distúrbios de comando de impulsos, assim como os desenvolvidos em jogos de azar em geral ou distúrbios patológicos, que podem influenciar diretamente no desempenho escolar, no trabalho, na convivência social, podendo resultar em problemas de saúde física, abrangendo visão nebulosa e dor nos pulsos ou atrás do pescoço.

Fortim e Araujo (2013) também ressaltam que a dependência de smartphone pode acarretar em prejuízos no trabalho, como faltas, mau rendimento, ou até mesmo atitudes que venham a colocar o colaborador em risco de demissão ou em circunstâncias desagradáveis pela falta de controle no uso. Os autores afirmam também que podem ser ocasionados danos financeiros na vida dos usuários e brigas no âmbito familiar, pois o uso contínuo desses aparelhos faz com que os seus usuários negligenciem o relacionamento com seus familiares, como cônjuge, pais, filhos e amigos que não são os virtuais.

Devido à falta de tempo, os consumidores possuem uma grande necessidade de conveniência, para que possam fazer o uso de seus smartphones em qualquer lugar e a qualquer momento (GENOVA, 2010). Lu e Su (2009) descrevem a conveniência e a praticidade dos smartphones como um fator positivo na vida de seus usuários, pois possibilitam aos seus donos a realização de transações em circunstâncias em que há restrições severas de tempo, como em um engarrafamento, ou na espera de alguém. Stephen e Davis (2009) afirmam que, devido a sua conveniência, o smartphone se tornou amplamente difundido entre os consumidores.

A natureza de dupla utilização dos smartphones gerou um aumento positivo no uso desses aparelhos e esta ampla difusão torna conveniente o seu uso (HAHN, 2010). Assim, os consumidores tornam-se dependentes dos smartphones, pela facilidade na obtenção de informações, navegação, entre outras (Genova, 2010), ou seja, o fator Conveniência está relacionado com o aumento da dependência do uso de smartphones.

Portanto, é apresentada a primeira hipótese proposta $(\mathrm{H} 1)$ :

Revista Eletrônica de Estratégia \& Negócios, Florianópolis, v.14, n. 1, jan./abr. 2021. 
H1: A conveniência exerce impacto positivo na dependência de uso de smartphones.

De acordo com Alter (2017), alguns indivíduos são mais obcecados pelo chamado "feedback social" do que outros. O autor também afirma que os humanos são seres sociais que não conseguem ignorar por completo o que outras pessoas pensam a seu respeito, e relata que a confirmação social, ou ver o mundo como os outros o veem, é uma forma de pertencer a um grupo de pessoas que pensam da mesma forma que a sua.

As necessidades sociais são as necessidades de interação social de um indivíduo, as quais representam o anseio de comunicar-se com seus amigos, família e demais pessoas ligadas ao seu grupo de convívio (TIKKANEN, 2009). Consumidores com uma alta necessidade social apresentam a necessidade de estar conectados, e, sobre isso, Bodker et al. (2009) afirma que a interação social vem sendo realizada via smartphone.

Logo, dentro desse contexto de socialização, o smartphone consiste em um aparelho que permite ao seu usuário a interação com outros usuários de forma mais eficiente e eficaz, sem limitações geográficas (CARAYANNIS; CLARCK; VALVI, 2012). Portanto, a necessidade social é um dos determinantes da dependência dos consumidores, pois os usuários podem manter-se conectados com outros indivíduos por meio do uso de seus smartphones (LIPPINCOTT, 2010). Sendo assim, acredita-se que os consumidores com um nível inferior de necessidades sociais deverão ter uma menor taxa de uso de smartphones (MORGAN, 2010). A partir do exposto, apresenta-se a segunda hipótese do estudo $(\mathrm{H} 2)$ :

H2: As necessidades sociais exercem impacto positivo na dependência de uso de smartphones.

A influência social pode ser definida como a forma de como as pessoas são afetadas pelas crenças, sentimentos e comportamentos dos outros indivíduos (MEl et al., 2012; MASON et al., 2007). Desse modo, os indivíduos muitas vezes não estão conscientes dos impactos das influências sociais (KLOBAS; CLYDE, 2001).

Os autores Kotler e Keller (2012) descrevem que o comportamento do consumidor é influenciado pelos fatores sociais, em que são levados em consideração os grupos de referência dos indivíduos. De acordo com os mesmos autores, nesses grupos, os indivíduos Revista Eletrônica de Estratégia \& Negócios, Florianópolis, v.14, n. 1, jan./abr. 2021. 
escolhem produtos que se comunicam com seus papéis sociais e status. Logo, ressalta-se a importância de os profissionais de marketing conhecerem esses potenciais status de produtos ou marcas.

Assim sendo, amigos e familiares podem exercer influência social sobre o uso de smartphones, acarretando incentivo a uma maior dependência de uso (AUTER, 2007). Dessa forma, os consumidores podem ser suscetíveis à influência social com relação aos smartphones pela observação, percepção ou antecipação de decisões tomadas pelas pessoas que fazem parte do seu convívio (SUKI, 2013). Sobre isso, Klobas e Clyde (2001) afirmam que as influências sociais podem exercer um impacto positivo sobre a dependência do uso de smartphones.

Nessa perspectiva, é apresentada a terceira (H3) hipótese:

H3: As influências sociais exercem impacto positivo na dependência de uso de smartphones.

De acordo com uma pesquisa realizada por Alter (2017, p. 25), “46\% das pessoas dizem que não suportam viver sem seus smartphones e $80 \%$ dos adolescentes verificam seus telefones pelo menos uma vez em uma hora". Conforme o autor, alguns usuários preferem sofrer danos físicos em si mesmos do que em seus smartphones. Esses são casos típicos da "nomofobia", o medo de estar sem o seu celular (traduzido pelas autoras).

Conforme Kim (2008), o comportamento de compra futura do consumidor será afetado por sua experiência passada. Por conseguinte, a experiência da dependência de smartphone pode ser positiva ou negativa, e, desta maneira, os consumidores vão ajustar rapidamente suas avaliações na compra de seu próximo smartphone (TING et al., 2011).

Isso posto, apresenta-se a quarta hipótese proposta pelo estudo (H4):

H4: A dependência de uso de smartphones exerce impacto positivo no comportamento de compra. 


\section{METODOLOGIA}

O estudo classificou-se como uma pesquisa de abordagem quantitativa (FONSECA, 2002) e de caráter descritivo (GIL, 2010). A pesquisa utilizou-se da técnica survey de coleta de dados, que, conforme Hair Jr. et al. (2005), é um procedimento de coleta de dados primários. Para essa técnica, os métodos de coleta de dados mais utilizados são os questionários, tradicionalmente usados para abordagens quantitativas, ou as entrevistas, para abordagens qualitativas.

Para atender aos objetivos da pesquisa, foi aplicado um questionário dividido em duas etapas, sendo a primeira com objetivo de identificar o perfil dos pesquisados, a partir das seguintes questões: idade, sexo, renda, etnia, estado civil, faixa etária, plano, operadora, marca e tempo de uso diário gasto utilizando o smartphone. A segunda etapa foi baseada no modelo teórico proposto no estudo, realizado por meio da escala Likert de 5 pontos, variando de $1=$ discordo totalmente a $5=$ concordo totalmente.

A população da pesquisa foi constituída por acadêmicos de cursos superiores de um Instituto Federal localizado na região sul do Brasil. De acordo com dados fornecidos pela instituição, o Instituto contava com 495 alunos matriculados nos cinco cursos superiores que formaram a população da atual pesquisa. Assim, a pesquisa foi realizada por meio de um procedimento de amostragem não probabilística e por conveniência, na qual o pesquisador escolhe sua população amostral (AAKER; KUMAR; DAY, 2011). Calculou-se que a amostra mínima da pesquisa fosse de 165 alunos, levando em consideração o parâmetro percentual estimado em 0,80 e erro amostral de 5\% conforme cálculo estatístico (HAIR JR et al.; 2005).

Antes de proceder à etapa de coleta dos dados, foi realizado um pré-teste em uma pequena amostra da população a ser estudada, com o intuito de verificar o correto entendimento das afirmativas, pois, assim como afirma Hair Jr. et al. (2005), nenhum questionário deve ser aplicado antes de ser avaliada a exatidão e a coerência das respostas. Em outros termos, os autores relatam que deve ser realizado um pré-teste em uma pequena amostra de respondentes, os quais devem possuir características semelhantes às da população alvo da pesquisa. 
Após a realização do pré-teste, foram efetuados os devidos ajustes e, posteriormente, foi efetuada a aplicação do questionário final em sala de aula durante o mês de outubro de 2018, mediante autorização dos professores. Após a aplicação do questionário, foi realizada a tabulação e a análise dos dados no software PASW Statistic21, sendo realizados os seguintes testes estatísticos: testes de frequências e percentagens, Alpha de Cronbach, estatísticas descritivas (médias e desvios-padrões) e análise de regressão linear. Desse modo, no intuito de facilitar a visualização, no Quadro 1 são apresentados os objetivos da pesquisa e os métodos estatísticos utilizados para atingi-los.

Quadro 1 - Objetivos da pesquisa e suas respectivas fases estatísticas

\begin{tabular}{|l|l|}
\multicolumn{1}{|c|}{ Objetivos da Pesquisa } & \multicolumn{1}{|c|}{ Métodos Estatísticos } \\
\hline $\begin{array}{l}\text { a) Identificar o perfil dos acadêmicos } \\
\text { b) Adaptar e validar o modelo de Ting et al. (2011) para o }\end{array}$ & Alfa de Cronbach \\
\hline $\begin{array}{l}\text { c) Mensurar a percepção dos pesquisados e a concordância das } \\
\text { respostas com relação à conveniência, necessidades sociais, } \\
\text { influências sociais, dependência de uso e comportamento de } \\
\text { compra }\end{array}$ & \multirow{2}{*}{$\begin{array}{l}\text { Estatísticas Descritivas (médias e desvios- } \\
\text { padrões) }\end{array}$} \\
$\begin{array}{l}\text { d) Testar o impacto e verificar se as necessidades sociais, } \\
\text { influência social e conveniência (variáveis independentes) são } \\
\text { antecedentes da dependência de uso de smartphone (variável } \\
\text { mediadora) }\end{array}$ & \multirow{2}{*}{ Análise de Regressão Linear } \\
\cline { 1 - 2 } $\begin{array}{l}\text { e) Mensurar o impacto da dependência de uso (variável } \\
\text { mediadora) no comportamento de compra (variável } \\
\text { dependente) }\end{array}$ & \\
\cline { 1 - 2 }
\end{tabular}

Fonte: Elaborado pelas autoras (2018).

\section{RESULTADOS E DISCUSSÕES}

Neste tópico são apresentadas as análises e discussões dos dados coletados.

\subsection{PERFIL DOS ACADÊMICOS}

Com o propósito de identificar o perfil dos pesquisados, foram realizados Testes de Frequências e percentagens, que, para Morettin e Bussab (2011), são testes que possuem o intuito de auxiliar o pesquisador a interpretar a proporção de cada variável em relação ao total destas variáveis. 
A amostra do estudo formou-se por 266 acadêmicos dos cursos superiores do Instituto Federal. Quanto ao sexo dos respondentes, os dados obtidos apontam que 148 $(55,6 \%)$ são do sexo feminino e 118 (44,4\%) são do sexo masculino, apontando uma predominância do sexo feminino nos resultados da pesquisa.

No que se refere à etnia (Gráfico 1) dos acadêmicos da amostra, foi constatado que 201 (75,5\%) respondentes são da etnia branca, 38 (14,3\%) da parda, 24 (9\%) da negra, 2 $(0,8 \%)$ da amarela e $1(0,4 \%)$ da indígena. Conforme dados do Censo Demográfico do IBGE (2010), 47,7\% da população brasileira possui a etnia branca, 43,3\% a etnia parda, 7,6\% preta, 1\% amarela e 0,4\% a etnia indígena. Em comparação com os dados obtidos na presente pesquisa, pode ser observado que a população da amostra encontra-se na mesma ordem que os dados do IBGE, ou seja, a etnia branca é a que apresenta as maiores percentagens, seguida da etnia parda, negra, amarela e indígena, respectivamente.

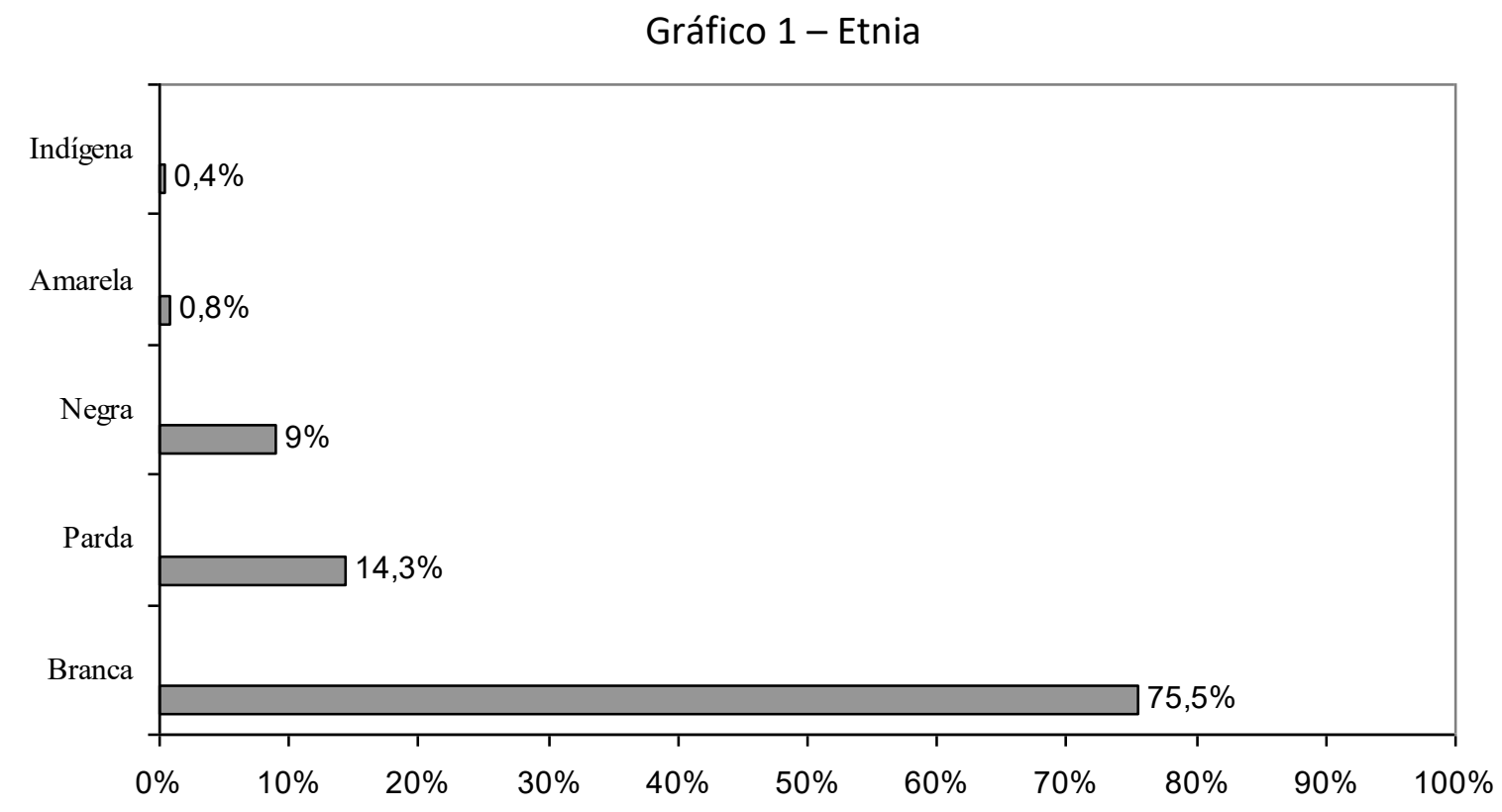

Fonte: Dados da pesquisa (2018).

Quanto à faixa etária dos respondentes, destaca-se a maior percentagem de 182 $(68,4 \%)$ acadêmicos com uma faixa de até 25 anos, e as menores percentagens foram 7 respondentes (2,6\%) de 41 a 45 anos e $6(2,4 \%)$ acima dos 45 anos. A menor idade relatada pelos respondentes foi 17 anos e a maior idade foi 54 anos. Esses dados podem ser verificados no Quadro 2. 
Quadro 2-Idade

\begin{tabular}{|c|c|c|}
\hline Faixa etária & Frequência & Percentual \\
\hline Até 25 anos & 182 & $68,4 \%$ \\
\hline De 26 a 30anos & 42 & $15,7 \%$ \\
\hline De 31 a 35 anos & 19 & $7,1 \%$ \\
\hline De 36 a 40 anos & 10 & $3,8 \%$ \\
\hline De 41 a 45 anos & 7 & $2,6 \%$ \\
\hline Acima de 45 anos & 6 & $2,4 \%$ \\
\hline
\end{tabular}

Fonte: Dados da pesquisa (2018).

Quanto à ocupação da amostra pesquisada, 153 (57,5\%) são estudantes e exercem alguma atividade profissional remunerada, $68(25,6 \%)$ somente estudam e $45(16,9 \%)$ são estudantes e realizam estágio, como pode ser observado no Quadro 3.

Quadro 3 - Ocupação

\begin{tabular}{|c|c|c|}
\hline Ocupação & Frequência & Percentual \\
\hline Estudante/Emprego & 153 & $57,5 \%$ \\
\hline Estudante/Estágio & 68 & $25,6 \%$ \\
\hline Somente Estudante & 45 & $16,9 \%$ \\
\hline
\end{tabular}

Fonte: Dados da pesquisa (2018).

No que se refere à renda total mensal dos pesquisados, a maior percentagem obtida foi de 145 (54,5\%) com renda de um a três salários mínimos, e a menor percentagem foi de 16 (6\%) que relataram ter renda total acima de sete salários mínimos. Tais dados encontram-se ilustrados no Quadro 4.

Quadro 4-Renda familiar

\begin{tabular}{|c|c|c|}
\hline Renda total & Frequência & Percentual \\
\hline Até 1 salário mínimo & 30 & $11,3 \%$ \\
\hline De 1 a 03 salários mínimos & 145 & $54,5 \%$ \\
\hline De 03 a 05 salários mínimos & 54 & $20,3 \%$ \\
\hline De 05 a 07 salários mínimos & 21 & $7,9 \%$ \\
\hline Acima de 07 salários mínimos & 16 & $6 \%$ \\
\hline
\end{tabular}

Fonte: Dados da pesquisa (2018).

Acerca do estado civil dos pesquisados, 218 (82\%) são solteiros, 24 (9\%) possuem união estável, $19(7,1 \%)$ são casados, 5 (1,9\%) são divorciados e 0 (0\%) declararam ser viúvos. Os dados dessa questão são apresentados no Quadro 5.

Quadro 5 - Estado civil

\begin{tabular}{|c|c|c|}
\hline Estado civil & Frequência & Percentual \\
\hline Solteiro (a) & 218 & $82 \%$ \\
\hline Casado (a) & 19 & $7,1 \%$ \\
\hline Divorciado (a) & 5 & $1,9 \%$ \\
\hline União estável & 24 & $9 \%$ \\
\hline
\end{tabular}

Revista Eletrônica de Estratégia \& Negócios, Florianópolis, v.14, n. 1, jan./abr. 2021. 


\begin{tabular}{|c|c|c|}
\hline Estado civil & Frequência & Percentual \\
\hline Viúvo (a) & 0 & $0 \%$ \\
\hline
\end{tabular}

Fonte: Dados da pesquisa (2018).

No que se refere à marca dos aparelhos, dos 256 entrevistados que afirmaram que possuem smartphone, destacam-se as percentagens dos 115 (45\%) acadêmicos que responderam que possuem um aparelho da marca Samsung, 50 (19,6\%) da Motorola, 41 (16\%) da LG, 27 (10,6\%) da Apple e os demais 23 (8,8\%) possuem outras marcas, como Asus, Alcatel, Sony, Lenovo, Microsoft, Blu, Quantum, Qbex, Xiaomi, Caterpilar ou Tp-link. Os dados podem ser observados no Gráfico 2.

\section{Gráfico 2 - Marca}

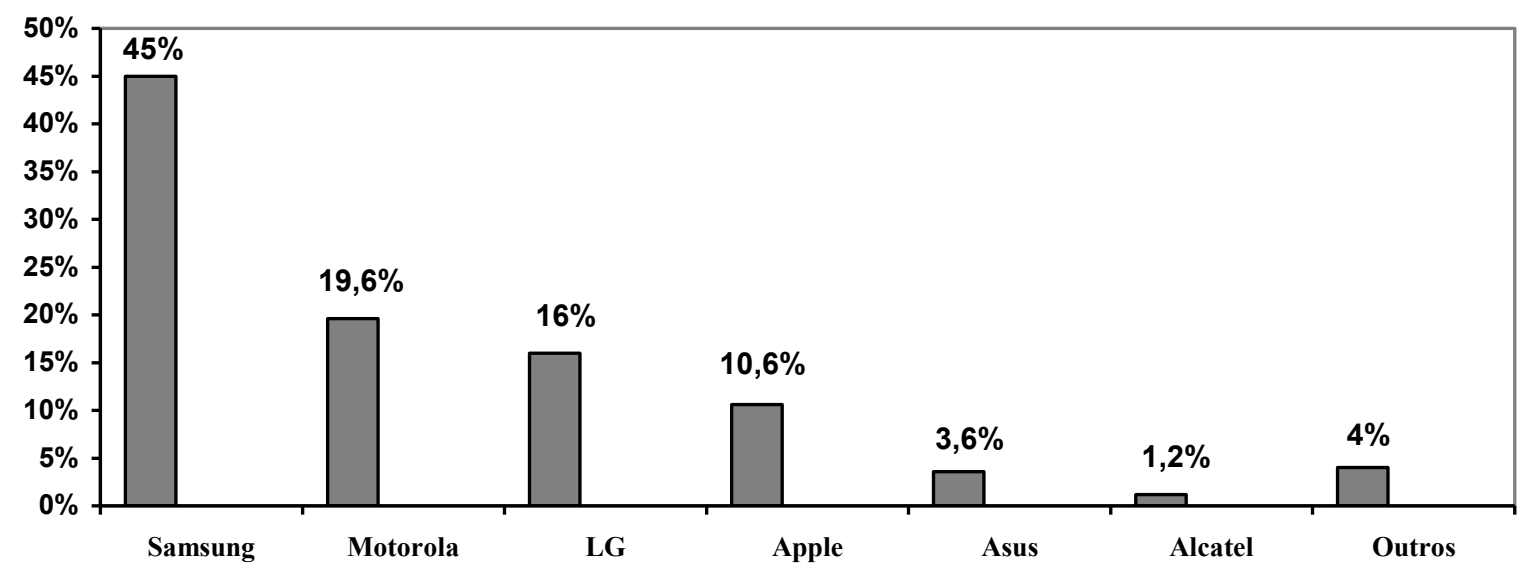

Fonte: Dados da pesquisa (2018).

Pode ser observado nos dados acerca da variável Marca, uma relação com a pesquisa da Universidade Martins do Varejo (UMV, 2018), a qual afirma que há quatro marcas que concentram o maior número de vendas de smartphones no Brasil: a Samsung, a Motorola, a LG e a Alcatel. No entanto, no caso dos dados da amostra do estudo, apenas a Alcatel atingiu uma média diferenciada da apresentada pela pesquisa da Universidade.

No quesito referente à operadora utilizada pelos pesquisados, podendo existir mais de uma resposta, as maiores médias obtidas foram: 225 (88\%) dos respondentes utilizando como primeira opção de operadora a Vivo e $11(4,2 \%)$ a operadora Oi. E como segunda opção de operadora, acrescentando a resposta da primeira opção, 15 respondentes afirmaram possuir mais de uma operadora em uso, sendo $1(6,7 \%)$ respondente possuindo a Vivo, $6(40 \%)$ a Oi e $8(53,3 \%)$ a Claro. Os dados desse quesito são apresentados no Gráfico 3. Revista Eletrônica de Estratégia \& Negócios, Florianópolis, v.14, n. 1, jan./abr. 2021. 
Gráfico 3 - Operadora

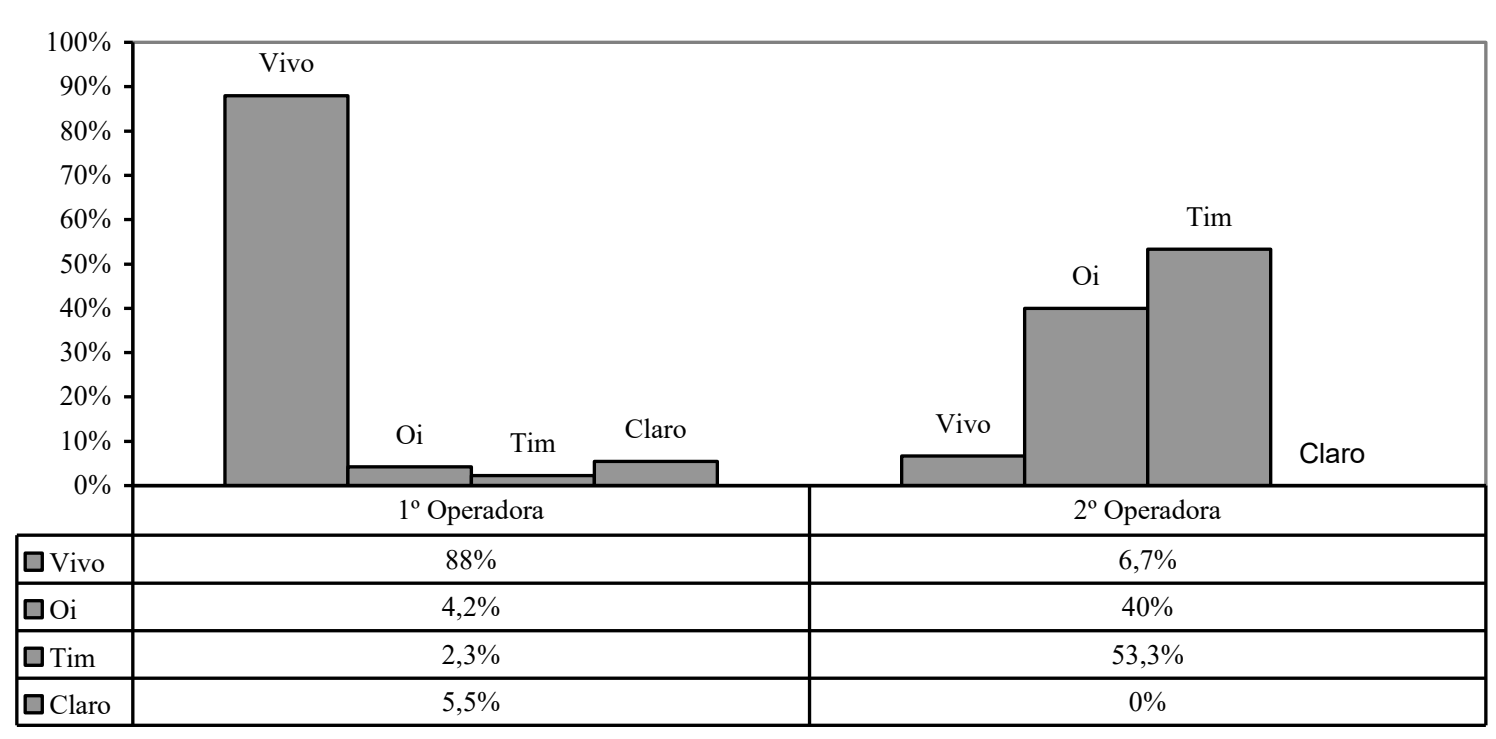

Fonte: Dados da pesquisa (2018).

O resultado desta variável pode ser relacionado com a pesquisa da Anatel (2018), a qual afirma que, no quesito Competição no ramo de telefonia móvel, a operadora Vivo possui uma participação de mercado bastante significativa em relação às demais operadoras.

Referente ao plano utilizado, foram obtidas respostas dos 266 acadêmicos, pelo fato de que, mesmo não possuindo um aparelho smartphone, os demais respondentes afirmaram possuir celulares. Assim, 181 (68\%) relataram que optam pelo plano Pré-Pago e 85 (32\%) pelo plano Pós-Pago. O Gráfico 4 expõe os dados acerca do plano.

\section{Gráfico 4 - Plano}

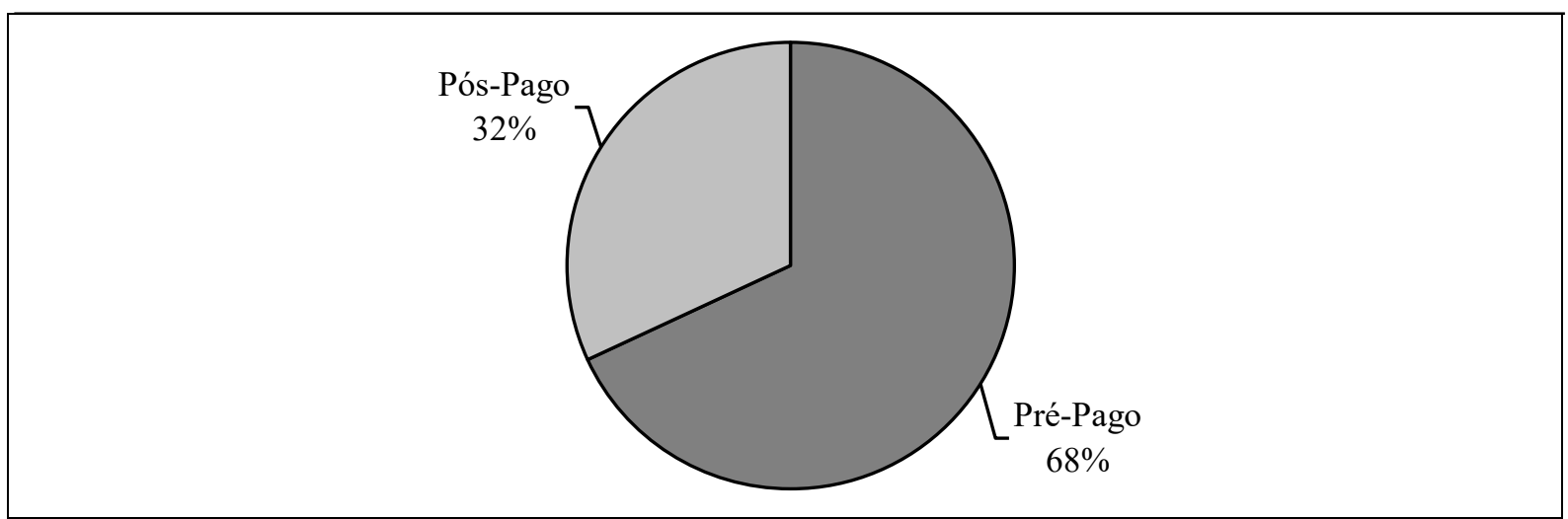

Fonte: Dados da pesquisa (2018).

Revista Eletrônica de Estratégia \& Negócios, Florianópolis, v.14, n. 1, jan./abr. 2021. 
Quanto aos dados encontrados na pesquisa referente ao plano, estes condizem com os dados do site da Anatel (2018), os quais demonstram que, das operadoras em atividade no Brasil, o número de smartphones e celulares habilitados no sistema Pré-pago é superior à quantidade de ativações de linhas Pós-pagas.

Quanto ao tempo de uso dos pesquisados com seus respectivos smartphones, a maioria dos respondentes, $103(38,7 \%)$, afirmou utilizar o aparelho em uma faixa de uma a três horas por dia, seguido de 72 (29,3\%) acadêmicos que afirmaram utilizar o aparelho por mais de 6 horas por dia. As demais percentagens podem ser visualizadas no Quadro 6.

Quadro 6-Tempo diário utilizando o smartphone

\begin{tabular}{|c|c|c|}
\hline Tempo de uso diário do Smartphone & Frequência & Percentual \\
\hline Não acesso diariamente & 6 & $2,3 \%$ \\
\hline Menos de 1 hora por dia & 17 & $6,4 \%$ \\
\hline Entre 1 a 3 horas por dia & 103 & $38,7 \%$ \\
\hline Entre 3 a 6 horas por dia & 62 & $23,3 \%$ \\
\hline Acima de 6 horas por dia & 72 & $29,3 \%$ \\
\hline
\end{tabular}

Fonte: Dados da pesquisa (2018).

Assim como foi apresentado anteriormente, de acordo com uma pesquisa realizada por Alter (2017, p. 25), "80\% dos adolescentes verificam seus telefones pelo menos uma vez em uma hora", o que pode ser relacionado com os resultados obtidos na pesquisa, na qual a grande maioria dos pesquisados afirmou acessar seu smartphone entre uma a três horas, entre três e seis horas e acima de seis horas, sendo o tempo total de uso diário. Logo, hipoteticamente, pode-se afirmar que os usuários da amostra verificam seus smartphones pelo menos uma vez em uma hora, assim como afirmou Alter (2017).

\subsection{ANÁLISE DE CONFIABILIDADE}

Com a finalidade de atender o segundo objetivo da pesquisa, que é adaptar e validar o modelo de Ting et al. (2011) para o contexto brasileiro, foi realizado o cálculo de Alfa de Cronbach, que, de acordo com Hair Jr. et al. (2005), é um método utilizado para medir a confiabilidade interna de itens em uma escala, e para obtenção do resultado é calculada a média dos coeficientes de todas as combinações possíveis das metades divididas. Ainda de acordo com o autor, um nível aceitável de confiabilidade significa que os respondentes estão 
respondendo às perguntas coerentemente. Na Tabela 1 é possível analisar as dimensões do Alfa de Cronbach.

Tabela 1 - Dimensão do coeficiente Alfa de Cronbach

\begin{tabular}{cc}
\hline Variação do coeficiente alfa & Intensidade da associação \\
\hline$<0,6$ & Baixa \\
$0,6 \mathrm{a}<0,7$ & Moderada \\
$0,7 \mathrm{a}<0,8$ & Boa \\
$0,8<0,9$ & Muito boa \\
0,9 & Excelente \\
\hline
\end{tabular}

Fonte: Hair Jr. et al (2005, p. 200).

Os resultados da pesquisa estão expostos na Tabela 2, onde pode ser observado que o maior Alfa encontrado foi na variável Necessidades Sociais, com $\alpha=0,78$, e, posteriormente, a variável Comportamento de Compra, com um $\alpha=0,709$, e a variável Dependência, com $\alpha=0,702$, todos apresentando uma intensidade de associação boa. As demais variáveis apresentaram uma intensidade moderada.

Tabela 2 - Alfa de Cronbach

\begin{tabular}{cc}
\hline Variável & Alfa de Cronbach \\
\hline Conveniência & 0,693 \\
Necessidades sociais & 0,786 \\
Influências sociais & 0,668 \\
Dependência & 0,702 \\
Comportamento de compra & 0,709 \\
\hline
\end{tabular}

Fonte: Dados da pesquisa (2018).

Portanto, os índices encontrados na pesquisa apresentaram valores superiores a 0,6 e 0,7 , o que indica que há consistência interna com intensidade de associação de moderada a boa entre todas as variáveis do modelo, ou seja, o modelo da pesquisa apresenta uma confiabilidade satisfatória entre todas as variáveis do construto proposto por Ting et al. (2011).

\subsection{TESTE DAS HIPÓTESES}

Com a finalidade de testar o impacto e verificar se as necessidades sociais, influência social e conveniência (variáveis independentes) são antecedentes da dependência de uso de smartphone (variável dependente) e mensurar o impacto da dependência de uso (variável independente) no comportamento de compra (variável dependente), foram realizadas análises de regressão linear, que, de acordo com Levine et al. (2012), são utilizadas para 
prever os valores de uma variável numérica dependente levando em consideração o valor de outras variáveis, as independentes.

Primeiramente, foi necessário estabelecer a correlação entre as variáveis independentes, em razão de que variáveis muito correlacionadas podem vir a prejudicar a capacidade do modelo, pois fornecem informações semelhantes que podem explicar a variável dependente (SILVER, 2000). A Tabela 3 ilustra a interpretação sobre a força de associação entre as variáveis com o coeficiente de correlação apresentado.

Tabela 3 - Níveis de correlação entre as variáveis

\begin{tabular}{cc}
\hline Coeficiente de correlação & Força de associação \\
\hline$(+-) 0.91-(+)) 1.00$ & Muito Forte \\
$(+-) 0.71-(+)) 0.90$ & Alta \\
$(+-) 0.41-(+)) 0.70$ & Moderada \\
$(+-) 0.21-(+-) 0.40$ & Pequena, mas definida \\
$(+-) 0.01-(+-) 0.20$ & Leve, quase imperceptível \\
\hline
\end{tabular}

Fonte: Adaptado de Hair Jr. et al. (2005).

No presente estudo, pode ser observado, no Quadro 7, que as variáveis independentes não apresentaram nenhuma correlação elevada. Portanto, como nenhuma correlação apresentou força de associação superior a 0,70 , é possível continuar com a análise de regressão, tendo em vista que nenhuma variável causa um fato de inflação de variação sobre a outra, ou seja, o modelo está livre de problemas de multicolineariedade.

Quadro 7 - Correlação das variáveis

\begin{tabular}{|c|c|c|c|c|c|}
\hline Variáveis & Conveniência & $\begin{array}{c}\text { Necessidades } \\
\text { Sociais } \\
\end{array}$ & $\begin{array}{c}\text { Influências } \\
\text { Sociais }\end{array}$ & Dependência & $\begin{array}{c}\text { Comportamento } \\
\text { de compra }\end{array}$ \\
\hline Conveniência & 1 & & & & \\
\hline $\begin{array}{c}\text { Necessidades } \\
\text { Sociais }\end{array}$ & 0,365 & 1 & & & \\
\hline $\begin{array}{l}\text { Influências } \\
\text { Sociais }\end{array}$ & 0,083 & 0,287 & 1 & & \\
\hline Dependência & 0,379 & 0,243 & 0,359 & 1 & \\
\hline $\begin{array}{c}\text { Comportamento } \\
\text { de compra }\end{array}$ & 0,355 & 0,560 & 0,139 & 0,347 & 1 \\
\hline
\end{tabular}

Fonte: Elaborado pelas autoras (2018).

Como pode ser observado no Quadro 8, a análise de Regressão linear aponta que dois (conveniência e influência social) dos três construtos do modelo impactam diretamente da variável Dependência de smartphones, pois apresentaram significância de 0,000 e 0,000, respectivamente, logo, Sig.< 0,05. Conforme estes resultados, não é possível aceitar a 
hipótese nula que estabelece a igualdade das variâncias. Já o construto Necessidade Social não apresenta impacto direto na dependência de smartphones, pois apresentou um resultado de significância de 0,666, logo, Sig > 0,05, e, portanto, é possível aceitar a hipótese nula.

Quadro 8-Coeficientes

\begin{tabular}{|c|c|c|c|c|c|c|c|}
\hline \multirow{2}{*}{ Modelo } & \multicolumn{2}{|c|}{$\begin{array}{l}\text { Coeficientes não } \\
\text { padronizados }\end{array}$} & \multirow{2}{*}{$\begin{array}{c}\text { Coeficientes } \\
\text { padronizados } \\
\text { Beta }\end{array}$} & \multirow{2}{*}{$\mathbf{t}$} & \multirow{2}{*}{ Sig. } & \multicolumn{2}{|c|}{$\begin{array}{c}\text { 95\% Intervalo de Confiança } \\
\text { para B }\end{array}$} \\
\hline & B & $\begin{array}{c}\text { Erro } \\
\text { estimado }\end{array}$ & & & & $\begin{array}{l}\text { Limite } \\
\text { inferior }\end{array}$ & Limite superior \\
\hline Constante & 0,659 & 1,283 & & 0,514 & 0,608 & $-1,867$ & 3,183 \\
\hline Conveniência & 0,504 & 0,085 & 0,343 & 5,965 & 0,000 & 0,338 & 0,671 \\
\hline $\begin{array}{l}\text { Necessidades } \\
\text { Sociais }\end{array}$ & 0,038 & 0,088 & 0,026 & 0,432 & 0,666 & 0,135 & 0,211 \\
\hline $\begin{array}{l}\text { Influências } \\
\text { Sociais }\end{array}$ & 0,462 & 0,080 & 0,323 & 5,782 & 0,000 & 0,305 & 0,619 \\
\hline
\end{tabular}

Fonte: Dados da pesquisa (2018).

Como pode ser observado no Quadro 9, a análise de regressão linear aponta que a variável Dependência de smartphones impacta no comportamento de compra, pois apresentou-se significância de 0,000, logo, Sig.< 0,05. Conforme este resultado, não é possível aceitar a hipótese nula que estabelece a igualdade das variâncias.

Quadro 9-Coeficientes

\begin{tabular}{|c|c|c|c|c|c|c|c|}
\hline \multirow{2}{*}{ Modelo } & \multicolumn{2}{|c|}{$\begin{array}{c}\text { Coeficientes não } \\
\text { padronizados }\end{array}$} & $\begin{array}{c}\text { Coeficientes } \\
\text { padronizados }\end{array}$ & \multirow{2}{*}{$\mathbf{t}$} & \multirow{2}{*}{ Sig. } & \multicolumn{2}{|c|}{ p5\% Intervalo de Confiança } \\
\cline { 2 - 4 } \cline { 6 - 8 } & B & Erro & Beta & & & $\begin{array}{c}\text { Limite } \\
\text { inferior }\end{array}$ & Limite superior \\
\hline Constimado & & & & 26,585 & 0,000 & 10,354 & 12,010 \\
\hline Dependência & 0,217 & 0,036 & 0,347 & 6,019 & 0,000 & 0,146 & 0,288 \\
\hline
\end{tabular}

Fonte: Dados da pesquisa (2018).

Desse modo, a análise de regressão apontou que a Conveniência e a Influência Social impactam diretamente na variável Dependência de smartphones. Já a variável Necessidade Social não apresentou um impacto direto na dependência de smartphones. Nestes termos, a conveniência e a influência social são antecedentes da dependência de uso de smartphone. Revista Eletrônica de Estratégia \& Negócios, Florianópolis, v.14, n. 1, jan./abr. 2021. 
Além disso, a variável Dependência de smartphones impacta no comportamento de compra, e, portanto, a dependência é antecedente do comportamento de compra.

\section{CONSIDERAÇÕES FINAIS}

Os smartphones tornaram-se indispensáveis à tecnologia e à conexão em si, e, assim como afirmam Ribeiro e Silva (2015), o uso dos smartphones tem apresentado um fator positivo no que se refere ao acesso de informações, comunicação, checagem de e-mails, redes sociais, mensagens, sites de notícias, etc. Entretanto, conforme é relatado pelos autores, o uso constante dessas tecnologias pode levar seus usuários a um ambiente propício ao uso abusivo e, possivelmente, a uma dependência desses aparelhos.

Além disso, um estudo realizado por Lin et al. (2013), aplicado em sala de aula, abrangendo 1.875 adolescentes do leste da Ásia, mostrou que os smartphones são altamente populares entre os adolescentes, sendo sua porcentagem de uso de quase $90 \%$ dos adolescentes da amostra. Outro resultado obtido pelo estudo foi de que os adolescentes utilizam seus smartphones majoritariamente para o lazer. Diante disso, a notável popularização desses aparelhos impulsionou a atual pesquisa a enriquecer a literatura sobre os fatores que impactam na dependência de smartphones, bem como a dependência impacta no comportamento de compra.

Sendo assim, quanto a sua natureza, a pesquisa realizada caracterizou-se como quantitativa e descrita, e quanto à coleta de dados, foi utilizada a técnica survey, com o intuito de investigar o impacto dos fatores Conveniência, Necessidades e Influências Sociais na dependência do uso de smartphones, bem como o impacto dessa dependência de uso no comportamento de compra de smartphones nos acadêmicos dos cursos superiores do Instituto Federal.

Referente ao primeiro objetivo proposto, por meio dos testes de frequências e percentagens, foi constatado que a maioria dos acadêmicos da amostra afirmou possuir um aparelho do tipo smartphone, sendo um total de 256 (96,2\%) dos respondentes; a marca mais utilizada é a Samsung, com 115 (45\%) afirmações; a operadora mais utilizada é a Vivo 
com 225 (88\%) acadêmicos; o plano mais presente nas respostas é o Pré-Pago, com 181 (68\%), e o gênero com maior predominância é o feminino, com 148 (55,6\%) afirmações.

Quanto à etnia dos pesquisados, a etnia com maior percentagem é a branca, com 201 $(75,5 \%)$ dos respondentes. Com relação à faixa etária, a resposta mais presente foi a de até 25 anos, com $182(68,4 \%)$ e, quanto à ocupação, mais da metade dos respondentes afirmaram que exercem atividade remunerada e estudam ao mesmo tempo, sendo 153 (57,5\%) afirmações.

No que se refere à renda dos pesquisados, a maioria relatou possuir uma renda total mensal de um a três salários mínimos, sendo 145 (54,5\%) dos respondentes. Quanto ao estado civil, a maior predominância foi de $81(30,4 \%)$ que afirmaram ser solteiros, e, por fim, referente ao tempo de uso dos smartphones, a maior percentagem obtida foi de 103 (38,7\%) dos acadêmicos que relataram utilizar estes aparelhos em uma faixa de uma a três horas por dia.

Como resposta ao segundo objetivo, por meio do Alfa de Cronbach, foi constatado que os índices encontrados na pesquisa apresentaram valores superiores a 0,6 e 0,7, o que indica que há consistência interna com intensidade de associação de moderada a boa entre todas as variáveis do modelo, ou seja, o modelo da pesquisa apresenta uma confiabilidade satisfatória entre todas as variáveis do construto proposto por Ting et al. (2011).

Referente ao quarto e ao quinto objetivo da pesquisa, foram realizadas análises de regressão linear, que apontaram que a conveniência e a influência social impactam diretamente na dependência de smartphones, o que confirma as hipóteses $\mathrm{H} 1$ e H3 do estudo. Já a variável Necessidade Social não apresentou um impacto direto na dependência de smartphones, e, sendo assim, a hipótese H2 não foi confirmada. Logo, a conveniência e a influência social são antecedentes da dependência de uso do smartphone. Por sua vez, a variável Dependência de smartphones apresentou impacto no comportamento de compra, e, sendo assim, a dependência é antecedente do comportamento de compra, confirmando, assim, a hipótese $\mathrm{H} 4$. 
Como contribuição do estudo, destaca-se contribuição prática, o qual pode servir de subsídio para a área de marketing das empresas do segmento de telefonia móvel, auxiliando os gestores na compreensão de como a conveniência e as necessidades sociais afetam a dependência de uso de smartphones, e como essa dependência afeta o comportamento de compra. Além disso, destaca-se a contribuição teórica para a ciência da Administração, que ocorreu por meio da adaptação e validação do modelo proposto por Ting et al. (2011) para o contexto brasileiro.

Diante de tais resultados, deve-se reforçar a importância do investimento no relacionamento com os consumidores e sugere-se uma reaplicação do modelo validado com outros públicos, pelo fato de que o presente estudo restringiu-se a uma amostra não probabilística dos acadêmicos de apenas um campus de um Instituto Federal, de modo que os resultados ficaram restritos a este tipo de público. Portanto, sugere-se que novas pesquisas sejam realizadas e comparadas, com os mais diversos tipos de públicos, visando conhecer melhor o comportamento dos consumidores das tecnologias móveis.

\section{REFERÊNCIAS}

AAKER, D. A.; KUMAR V.; DAY, G. S. Pesquisa de Marketing. Traduzido por: Reynaldo Cavalheiro Marcondes. 2. ed. São Paulo: Atlas, 2011.

ALTER, A. Irresistible: The Rise of Addictive Technology and the Business of Keeping Us Hooked. Hardcover: 2017.

ANATEL. Telefonia Móvel: Acessos (2018). Disponível em: <http://www.anatel.gov.br/dados/destaque-1/283-brasil-tem-236-2-milhoes-de-linhasmoveis-em-janeiro-de-2018> Acesso em: 27 mai. 2018.

AUTER, P. J. Portable social groups: will ingness to communicate, interpersonalcommunication gratifications, and cellphone use among young adults.International Journal Mobile Communications, v. 15, n. 2, p.139-56. 2007.

BODKER, M.; GIMPEL, G.; HEDMAN, J. Technology use as consumer: a longitudinal study of telefones inteligentes, Trigésima Conferência Internacional sobre Sistemas de Informação, Phoenix, Vol. 88, pp. 1-7, 2009.

BORGES, A. P; JOIA, L. A. Executivos e Smartphones: Uma relação ambígua e paradoxal. Revista Organizações e Sociedade, v. 20, n. 67, p.585-602, 2013. 
CARAYANNIS, E. G.; CLARK, S. C.; VALVI, D. E. Smartphone affor dance: achieving better business through innovation. Journal of the Knowledge Economy, v. 22, n. 6, p.1-11, 2012.

CHURCHILL, G. A.; PETER, J. P. Marketing: criando valor para os clientes. 2. ed. São Paulo: Saraiva, 2010.

COBRA, M. Administração de marketing no Brasil. 3.ed. Rio de Janeiro: Elsevier, 2014.

CORRÊA, H. L.; CAON, M. Gestão de serviços: lucratividade por meio de operações e de satisfação dos clientes. São Paulo: Atlas, 2002.

FONSECA, J. J. S. Metodologia da pesquisa científica. Fortaleza: UEC, 2002. Apostila.

FORTIM, I; ARAUJO, C. A. Aspectos psicológicos do uso patológico de internet. 2013. Tese (Doutorado em Psicologia Clínica) - Pontifícia Universidade Católica de São Paulo, São Paulo, V. 33, no 85, p. 292-311, 2013.

FUNDAÇÃO GETÚLIO VARGAS (FGV). 29ạ Pesquisa Anual do Uso de TI, 2018. Disponível em: <https://eaesp.fgv.br/sites/eaesp.fgv.br/files/pesti2018gvciappt.pdf> Acesso em: 16 jun. 2021.

FUTRELL, C. M. Vendas: o guia completo. 12. ed. Porto Alegre: AMGH, 2014.

GENOVA, G. L. The anywhere office - anywhere liability. Business Communication Quarterly, v. 73, n. 1, p. 119-26, 2010.

GIL, A. C. Como elaborar projetos de pesquisa. 5. ed. Atlas: São Paulo, 2010.

GREWAL. D.: LEVY, M. Marketing. 2.ed. Bookman, Porto Alegre, 2012.

HAHN, J. "Information seeking with Wikipedia on iPod Touch", Reference Services Review, v. 38, n. 2, p. 284-98, 2010.

HAIR Jr, J. F. et al. Fundamentos de métodos de pesquisa em administração. Porto Alegre: Bookmann, 2005.

INSTITUTO BRASILEIRO DE GEOGRAFIA E ESTATÍ́sTICA [IBGE]. Pesquisa Nacional por Amostra de Domicílios Contínua (PNAD), 2018. Disponível em:

<https://biblioteca.ibge.gov.br/visualizacao/livros/liv101543.pdf>. Acesso em: 24 abr. 2018.

INSTITUTO BRASILEIRO DE GEOGRAFIA E ESTATÍ́STICA [IBGE]. Tabela 3175 -População residente, por cor ou raça, segundo a situação do domicílio, o sexo e a idade, 2010. Disponível em: < https://sidra.ibge.gov.br/tabela/3175>. Acesso em 22 nov. 2018.

KLOBAS, J. E.; CLYDE, L. A. Social Influence and Internet use, Library Management, Vol. 22, No. 1, pp. 61-7., 2001. 
$\mathrm{KIM}, \mathrm{S}$. H. Moderating effects of job relevance and experience on mobile wireless technologyacceptance: adoption of a smartphone by individuals. Information and Management, v. 45, p. 387-93, 2008.

KOTLER, P.; KELLER, K. L. Administração de Marketing. 14. ed. Pearson: São Paulo, 2012.

LAS CASAS, A. L. Marketing: conceitos, exercícios, casos. 9. ed. São Paulo: Atlas, 2017.

LEMOS, A. Comunicação e práticas sociais no espaço urbano: as características dos Dispositivos Híbridos Móveis de Conexão Multirredes (DHMCM). Revista Comunicação, Mídia e Consumo, número 10, ESPM, São Paulo, 2007.

LEVINE, D. M. et al. Estatística: teoria e aplicações: usando Microsoft ${ }^{\circledR}$ Excel em Português. 6. ed. LTC, 2012.

LIN, Wan-Ying et al. From the wired to wireless generation? Investigating teens' Internet use through the mobile phone. Telecommunications Policy, v. 37, n. 8, p. 651-661, 2013.

LIPPINCOTT, J. K. A mobile future for academic libraries. Reference Services Review, v.38, n. 2, p.1-20, 2010.

LU, H-P. and SU, P.Y-J. "Factors affecting purchase intention on mobile shopping web sites", Internet Research, v. 19, n. 4, p. 442-58, 2009.

MASON, W. A.; CONREY, F. D.; SMITH, E. R. Situando processos de influência social: dinâmica, fluxos multidirecionais de influência nas redes sociais, Revisão da Personalidade e da Psicologia Social, Vol. 11, pp. 279-300, 2007.

$\mathrm{MEI}, \mathrm{M}$. et al. Conceptual paper: factors affecting the demand of smartphone among Young adult. International Journal on Social Science Economics and Art, v. 10, n. 2, p. 332-4, 2012.

MOK, J. Y. et al. Latent class analysis on internet and smartphone addiction in college students. Dovepress, v. 10, p. 817-828, 2014.

MORETTIN, Pedro Alberto; BUSSAB, Wilton de Oliveira. Estatística básica. 7. ed. rev. e atual. São Paulo: Saraiva, 2011.

MORGAN, J. J. Social networking websites teaching appropriate social competence to students with emotional and behavioral disorders. Intervention in School and Clinic, v. 45, n. 3, p. 147-5, 2010.

PICON, F. et al. Precisamos falar sobre tecnologia: caracterizando clinicamente os subtipos de dependência de tecnologia. Revista Brasileira de Psicoterapia, v. 17, n. 2, p. 44-60, 2015.

RIBEIRO, Q; SILVA, R. B. A. R. Os impactos dos dispositivos móveis nas pessoas. Revista Fatec Zona Sul, v. 2, n.1, 01-21, 2015. 
ROCHA, A. V. F et al. Dependência Digital: Dá pra viver desconectado? In: EXPOSIÇÃO DA PESQUISA EXPERIMENTAL EM COMUNICAÇÃO, Uberlândia, 2015. Anais. Uberlândia:

Sociedade Brasileira de Estudos Interdisciplinares da Comunicação, 2015.

SILVER, Mick. Estatística para administração. São Paulo: Atlas, 2000.

STEPHEN, K. K.; DAVIS, J. The social influences on electronic multitarefa in organization reuniões, Management Communication Quarterly, Vol. 23 No. 1, pp. 63-83, 2009.

SUKI, N. M. Students' dependence on smart phones. Campus-Wide Information Systems, v. 30, n. 2, p. $124-134,2013$.

TIKKANEN, I. Hierarquia de Maslow e sugestões dos alunos para o desenvolvimento de refeições escolares, Nutrição and Food Science, Vol. 39 No. 5, pp. 534-43, 2009.

TING, et al. "Dependency on smartphone and the impact on purchase behavior", Young Consumers, Vol. 12 Iss 3 pp. 193 - 203. 2011.

UNIVERSIDADE MARTINS DO VAREJO (UMV), Quatro marcas concentram as vendas de celulares no Brasil. Disponível em: <http://umv.com.br/quatro-marcas-concentram-asvendas-de-celulares-no-brasil/>. Acesso em: 31 mai. 2018. 